\title{
The Variable $\lambda$ Method of Sedimentation Equilibrium Applied to Polychloroprene and Polyisobutylene
}

\author{
Satoshi Itou, Yoshiyuki Einaga, and Hiroshi Fujita \\ Department of Polymer Science, Osaka University, \\ Toyonaka, Osaka 560, Japan.
}

(Received December 11, 1975)

\begin{abstract}
The variable $\lambda$ sedimentation equilibrium method for determining average molecular weights of polymeric substances was applied to narrow-distribution fractions of polychloroprene (PCP) and polyisobutylene (PIB) and a broad-distribution sample of PCP. The solvents used were methyl ethyl ketone (MEK) at $25.0^{\circ} \mathrm{C}$ for PCP and ethyl- $n$-heptanoate $(\mathrm{EH})$ at $34.5^{\circ} \mathrm{C}$ for PIB; both are $\theta$ solvents for the respective polymers. Values of $\bar{M}_{n}, \bar{M}_{w}$, and $\bar{M}_{z}$ were obtained with certainty, and those of the former two agreed closely with the results from other methods. No reasonable estimate of $\bar{M}_{z+1}$ was feasible. From comparisons with the theoretical prediction, the narrowdistribution fractions studied were found to be still far from monodisperse in molecular weight. The variable $\lambda$ method may be a sensitive means for the detection of molecular weight heterogeneity when the polymer sample is close to monodispersity.

KEY WORDS Sedimentation Equilibrium / Variable $\lambda$ Method / Average Molecular Weights / Polychloroprene / Polyisobutylene / Theta Solvents /
\end{abstract}

In 1960 one of the present authors (H.F.) ${ }^{1}$ described the theory for a novel type of sedimentation equilibrium experiment, which he later called the variable $\lambda$ method. ${ }^{2}$ This method predicted that if one were to work with pseudoideal solutions (ones in which the second virial coefficient $A_{2}$ vanishes), successive average molecular weights $\bar{M}_{n}, \bar{M}_{w}, \bar{M}_{z}, \cdots$ of a polymer could be determined from a series of sedimentation equilibrium measurements in which a parameter $\lambda$ was varied at suitable intervals over a wide range, theoretically from zero to infinity. Here $\lambda$ is defined by

$$
\lambda=\left(r_{\mathrm{b}}{ }^{2}-r_{\mathrm{m}}{ }^{2}\right)\left(1-\bar{v} \rho_{0}\right) \omega^{2} /(2 R T)
$$

where $\omega$ is the angular speed of the rotor, $r_{\mathrm{m}}$ and $r_{\mathrm{b}}$ are the radial distances from the center of rotation to the air-liquid meniscus and the bottom of a solution column in the ultracentrifuge cell, $\bar{v}$ is the partial specific volume of the solute polymer, $\rho_{0}$ is the solvent density, and $R T$ has the usual meaning. This parameter may be changed experimentally by varying the rotor speed and/or the depth of the solution column $r_{\mathrm{b}}-r_{\mathrm{m}}$.

Osterhoudt and Williams ${ }^{3}$ were the first to apply the variable $\lambda$ method to an actual system, but the potential of the method for the evaluation of $\bar{M}_{n}$ was left unexplored since their measurements were restricted to relatively low $\lambda$. Recently, Scholte $^{4}$ has extended the original formulation of Fujita and tested the method with extensive measurements on polyethylene, polystyrene, and polycaprolactam. The results obtained demonstrated the accuracy and limitations of the variable $\lambda$ method. In brief, Scholte's work found for $\bar{M}_{n}, \bar{M}_{w}$, and $\bar{M}_{z}$ values which compared favorably with those from other equilibrium measurements, but failed to obtain reliable values for $\bar{M}_{z+1}$. The higher average molecular weights were utterly inaccessible to experimental determination.

The present paper is concerned with further tests of the variable $\lambda$ method. The polymer samples chosen were narrow-distribution fractions of polychloroprene (PCP) and polyisobutylene (PIB) and a broad-distribution fraction of PCP. We were especially interested in work with narrow-distribution samples, because the previous authors mentioned had concerned themselves with relatively broad-distribution ones and also because there has been an increased interest in the 
molecular characterization of samples approaching monodispersity.

For PCP there is available a $\theta$ solvent very convenient for experimental work. It is methyl ethyl ketone (MEK) at $25.0^{\circ} \mathrm{C}$. The result is due to Hanafusa, et al., ${ }^{5}$ who showed that the buoyancy factor $1-\bar{v} \rho_{0}$ and the specific refractive index increment $\mathrm{d} n / \mathrm{d} c$ for PCP in this $\theta$ solvent were large enough for ultracentrifugal measurements to be made. All the present measurements on PCP were thus performed in MEK at $25.0^{\circ} \mathrm{C}$. On the other hand, none of the known $\theta$ solvents for PIB were appropriate for a sedimentation experiment. They gave too small a value for the buoyancy factor and/or the refractive index increment, or the $\theta$ temperatures were too high for our ultracentrifuge. After much consideration we finally decided to carry out measurements on PIB with ethyl- $n$-heptanoate $(E H)$ as solvent. For it was found from light-scattering measurements that the system PIB-EH had a $\theta$ temperature at $34.5^{\circ} \mathrm{C}$, a convenient location for ultracentrifugation. Note that this $\theta$ temperature is $1.5^{\circ} \mathrm{C}$ higher than the one reported by Fox and Flory, ${ }^{6}$ who estimated it from viscosity data. As shown below, the refractive index increment for PIB in EH was moderately large, at least sufficient for optical measurements of the sedimentation patterns. The problem was that the buoyancy factor of this system was as small as 0.058. Our attempt to use PIB even with this decisive disadvantage was undertaken because it became urgent to establish a means for an absolute determination of $\bar{M}_{z}$ of this polymer in conjunction with another project in our laboratory.

\section{EXPERIMENTAL}

\section{Polymer Samples}

The narrow- and broad-distribution fractions of PCP studied are designated by the letters $f$ and $F$, respectively. They were extracted by extensive fractionation from a Denka-Chloroprene of $\bar{M}_{v}=13 \times 10^{4}$, which had been specially prepared at low conversion by Denkikagaku Kogyo Co., Japan. For PIB we chose one of the narrow-distribution fractions previously subjected to a detailed solution study by Matsumoto, et $a l{ }^{7} \quad$ These authors reported $\bar{M}_{n}=15.3 \times 10^{4}$ and
$\bar{M}_{w}=16.0 \times 10^{4}$ for the chosen fraction.

\section{Buoyancy Factor}

The value of 0.382 , obtained previously by Hanafusa, et al. ${ }^{5}$ was assigned to the buoyancy factor of $\mathrm{PCP}$ in $\mathrm{MEK}$ at $25.0^{\circ} \mathrm{C}$. The corresponding value for the system PIB-EH at $34.5^{\circ} \mathrm{C}$ was found to be $0.058_{4}$ from density measurements with a pycnometer of about $30-\mathrm{m} l$ capacity. No great accuracy can be claimed for this value.

\section{Refractive Index Increment}

Murakami, et al., ${ }^{8}$ had reported a value of $0.154 \mathrm{ml} / \mathrm{g}$ for the $\mathrm{d} n / \mathrm{d} c$ of PCP in MEK at $25.0^{\circ} \mathrm{C}$. This value was used for the present data analysis as well. PIB in $\mathrm{EH}$ at $34.5^{\circ} \mathrm{C}$ we obtained a value of $0.102 \mathrm{ml} / \mathrm{g}$ at the wavelength $546 \mathrm{~nm}$ from measurements with a differential refractometer of the Schulz-Cantow design.

\section{Sedimentation Equilibrium Measurement}

A Beckman-Spinco analytical ultracentrifuge equipped with an electronic speed control system was used. Most of the sedimentation patterns were photographed by the schlieren optical system. For a given initial concentration the rotor speed was increased or decreased in stepwise fashion, after the attainment of sedimentation equilibrium had been checked at each step. In general, higher rotor speeds were chosen for lower initial concentrations. In this way, we obtained from measurements on solutions of differing concentrations groups of data points which, when combined, covered a wide range of the parameter $\lambda$. The concentration ranges studied were up to about $0.6 \mathrm{~g} / \mathrm{d} l$ for the system PCP-MEK and to about $0.75 \mathrm{~g} / \mathrm{d} l$ for the system PIB-EH. Al-double sector and Kel-F-double sector cells of $12 \mathrm{~mm}$ high were used, depending on the rotor speed chosen. The solution columns used ranged from 1.3 to $2.6 \mathrm{~mm}$ in depth for PCP-MEK and from 1.5 to $2.2 \mathrm{~mm}$ in depth for PIB-EH. Sedimentation equilibria were attained in intervals of time which were virtually independent of rotor speed and initial concentration. For example, when the column was about $2.0 \mathrm{~mm}$ deep, the times required were about $8 \mathrm{hr}$ for PCP-MEK and about $20 \mathrm{hr}$ for PIB-EH. There were no substantial differences in the rate of attainment of equilibrium between two successive steps in a series of experiments 
with varying rotor speed.

Heights of each schlieren curve above the base line at three points defined by $\xi=1 / 4,1 / 2$, and $3 / 4$ were read on a Nikkon Shadowgraph Model 6. Here $\xi$ is a reduced cell coordinate defined by

$$
\xi=\left(r^{2}-r_{\mathrm{m}}{ }^{2}\right) /\left(r_{\mathrm{b}}{ }^{2}-r_{\mathrm{m}}{ }^{2}\right)
$$

with $r$ being the radial distance from the center of rotation to a particular point in the solution column. Blurring of the edges of the photographed curves often made it difficult to locate their midpoints with accuracy, and thus introduced errors into the determinations of the $q$ functions that appear below. The measured heights of the schlieren curves were converted into the concentration gradients $\mathrm{d} c / \mathrm{d} r$ and then into $\mathrm{d} c / \mathrm{d} \xi$ in terms of the $\mathrm{d} n / \mathrm{d} c$ values and the instrument constant of the ultracentrifuge used. The last quantity was determined for bar angles of 75 and $80^{\circ}$ from measurements with aqueous sucrose solutions of $25.0^{\circ} \mathrm{C}$.

\section{Osmotic Pressure Measurement}

The $\bar{M}_{n}$ of the PCP fractions $f$ and $F$ were evaluated from osmotic pressure measurements on their MEK solutions at $30.0^{\circ} \mathrm{C}$. Use was made of a Knauer osmometer, together with adequately conditioned Sartorius Membranfilter Ultracellafilters ff and fff.

\section{RESULTS AND DISCUSSION}

\section{Summary of Basic Relations}

We define a quantity $q$ by

$$
q=\left(\lambda c_{0}\right)^{-1}(\mathrm{~d} c / \mathrm{d} \xi)
$$

where $c_{0}$ is the initial concentration of a given polymer solution which is to be subjected to a sedimentation experiment, $\lambda$ is the parameter defined by eq 1 , and $\mathrm{d} c / \mathrm{d} \xi$ is the concentration gradient at sedimentation equilibrium defined in terms of the reduced cell coordinate $\xi$ (see eq 2). In general, $q$ is a function of $c_{0}, \xi$, and $\lambda$. For pseudoideal solutions, however, the dependence on the first disappears, ${ }^{2}$ and $q$ may then be written $q_{\xi}(\lambda)$. It is to be noted that $q$ is an experimentally measurable quantity.

The following set of relations can be derived from the equation descriptive of sedimentation equilibrium of a polydisperse homopolymer in a single solvent. ${ }^{1-4}$

$$
\begin{gathered}
\bar{M}_{n}=16.83 / \int_{0}^{\infty} \lambda q_{1 / 2}(\lambda) \mathrm{d} \lambda \\
\bar{M}_{n}=5.31 / \int_{0}^{\infty} \lambda q_{1 / 4}(\lambda) \mathrm{d} \lambda \\
\bar{M}_{w}=\lim _{\lambda \rightarrow 0} q_{1 / 2}(\lambda)=\lim _{\lambda \rightarrow 0} q_{1 / 4}(\lambda) \\
\bar{M}_{w}=\lim _{\lambda \rightarrow 0}(1 / 2)\left[q_{1 / 4}(\lambda)+q_{3 / 4}(\lambda)\right] \\
\bar{M}_{w} \bar{M}_{z}=2 \lim _{\lambda \rightarrow 0}(\mathrm{~d} / \mathrm{d} \lambda)\left[q_{3 / 4}(\lambda)-q_{1 / 4}(\lambda)\right] \\
\bar{M}_{w} \bar{M}_{z} \bar{M}_{z+1}=-48 \lim _{\lambda \rightarrow 0}\left(\mathrm{~d} / \mathrm{d} \lambda^{2}\right)\left[q_{1 / 4}(\lambda)+q_{3 / 4}(\lambda)\right] \\
\bar{M}_{w} \bar{M}_{z} \bar{M}_{z+1}=-24 \lim _{\lambda \rightarrow 0} \mathrm{~d} q_{1 / 2}(\lambda) / \mathrm{d} \lambda^{2}
\end{gathered}
$$

These relations allow the average molecular weights $\bar{M}_{n}, \bar{M}_{w}, \bar{M}_{z}$, and $\bar{M}_{z+1}$ to be determined from measurements of $q_{1 / 4}, q_{1 / 2}$, and $q_{3 / 4}$ as functions of $\lambda$. For $\bar{M}_{n}$ the data must be obtained over the range of $\lambda$ from zero to a value at which either $\lambda q_{1 / 4}$ or $\lambda q_{1 / 2}$ becomes sufficiently close to zero. On the other hand, evaluation of the higher average molecular weights requires only the data at smaller $\lambda$, but such data must be of high accuracy, particularly for the use of eq 9 and 10. However, this requirement is not always met in practice, because, as can be seen from eq 3 , the concentration gradient diminishes with the decrease in $\lambda$ if $q$ is to remain finite at the limit of vanishing $\lambda$.

It can be shown ${ }^{2,4}$ that $q_{1 / 4}(\lambda)$ and $q_{1 / 2}(\lambda)$ satisfy the relations

$$
\begin{aligned}
& \int_{0}^{\infty} q_{1 / 4}(\lambda) \mathrm{d} \lambda=2.64 \\
& \int_{0}^{\infty} q_{1 / 2}(\lambda) \mathrm{d} \lambda=4.93
\end{aligned}
$$

regardless of molecular weight heterogeneity of the sample. This fact provides a useful means of checking the accuracy of the experimental data obtained.

\section{POLYCHLOROPRENE}

\section{Molecular Weights}

Figure 1 shows plots of $q_{1 / 2}$ and $q_{1 / 4}$ as functions of $\lambda$ for the PCP fraction $f$ in MEK at $25.0^{\circ} \mathrm{C}$. The corresponding plots for the PCP fraction $F$ are presented in Figure 2. In each 


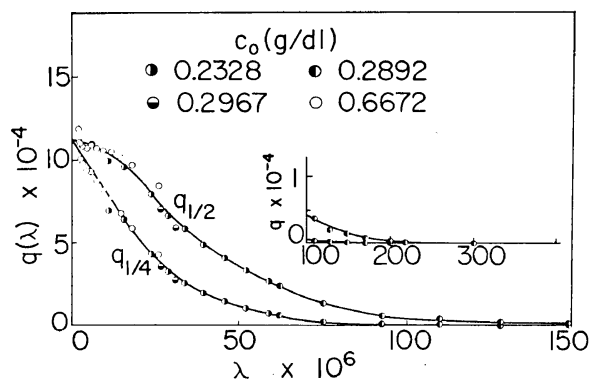

Figure 1. $q_{1 / 2}(\lambda)$ and $q_{1 / 4}(\lambda)$ vs. $\lambda$ for PCP (polychloroprene) fraction $f$ in MEK (methyl ethyl ketone) at $25.0^{\circ} \mathrm{C}$. The plots at larger $\lambda$ are enlarged in the insert. Various types of circles correspond to different initial concentrations $c_{0}$.

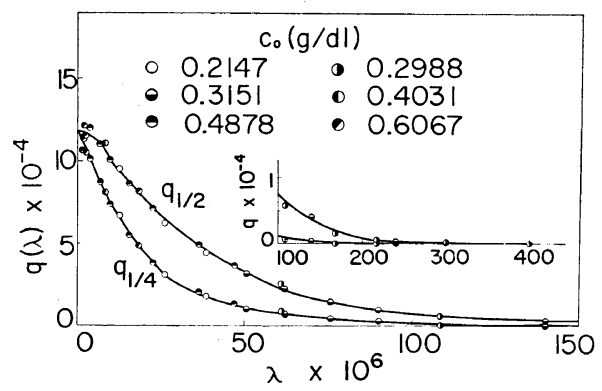

Figure 2. $q_{1 / 2}(\lambda)$ and $q_{1 / 4}(\lambda)$ vs. $\lambda$ for PCP fraction $F$ in $\mathrm{MEK}$ at $25.0^{\circ} \mathrm{C}$. The plots at larger $\lambda$ are enlarged in the insert. Various types of circles correspond to different initial concentrations $c_{0}$.

of the graphs, the data for different initial concentrations $c_{0}$ are distinguished by different marks. Thus, a group of points of the same mark contains data for varying rotor speeds and/or heights of the solution column. In agreement with the theoretical prediction, these groups of data points for differing $c_{0}$ form a single continuous curve, with no significant scattering. This behavior was not touched upon by Scholte, ${ }^{4}$ who determined the $q$ functions only at a single concentration. Applying eq 6 to Figures 1 and 2, we find $\bar{M}_{w}=(11.2 \pm 0.2) \times 10^{4}$ for the fraction $f$ and $\bar{M}_{w}=(11.8 \pm 0.2) \times 10^{4}$ for the fraction $F$.

The areas under the thick solid lines in Figure 1 are 4.87 and 2.63 for $q_{1 / 2}$ and $q_{1 / 4}$, respectively. The former is about $1.5 \%$ smaller than the theoretical value 4.93 , whereas the latter virtually agrees with the theoretical one 2.64. The corresponding figures for the fraction $F$ are 4.83

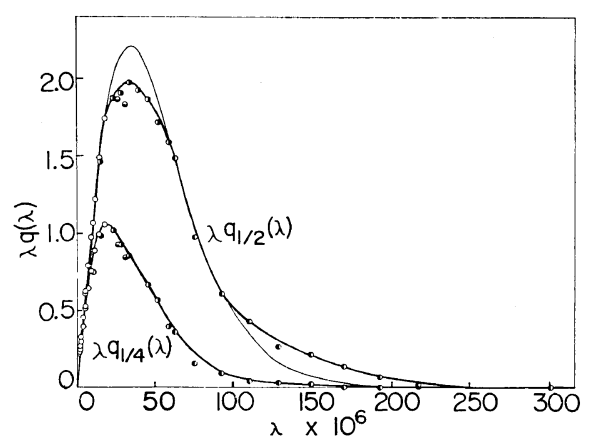

Figure 3. $\lambda q_{1 / 2}(\lambda)$ and $\lambda q_{1 / 4}(\lambda) v s$. $\lambda$ for PCP fraction $f$ in MEK at $25.0^{\circ} \mathrm{C}$. The circles have the same meaning as in Figure 1. Thin line: $\lambda q_{1 / 2}(\lambda)$ calculated from eq 13 for a monodisperse sample with $M=11.2 \times 10^{4}$.

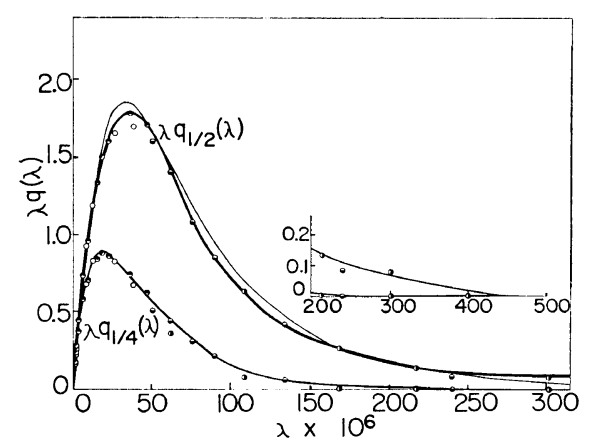

Figure 4. $\lambda q_{1 / 2}(\lambda)$ and $\lambda q_{1 / 4}(\lambda) v s$. $\lambda$ for PCP fraction $F$ in $\mathrm{MEK}$ at $25.0^{\circ} \mathrm{C}$. The plots at larger $\lambda$ are enlarged in the insert. The circles have the same meaning as in Figure 2. Thin line: $\lambda q_{1 / 2}(\lambda)$ calculated from eq 13,14 , and 15 for a polydisperse sample with a logarithmic normal type of molecular weight distribution.

and 2.59 , which check the theoretical predictions somewhat less satisfactorily. It seems that errors of these magnitudes approach the limits of accuracy of the schlieren optical system.

Figures 3 and 4 display plots of $\lambda q_{1 / 2}$ and $\lambda q_{1 / 4}$ against $\lambda$ constructed from Figures 1 and 2. It is to be observed that for either sample these plots can be extended without much arbitrariness to the asymptotic zero level. This feature is essential for the use of eq 4 or eq 5 . Applying these equations to Figure 3, we find $\bar{M}_{n}=10.7 \times 10^{4}$ from the curve for $\lambda q_{1 / 2}$ and $\bar{M}_{n}=10.3 \times 10^{4}$ from the curve for $\lambda q_{1 / 4}$. The 


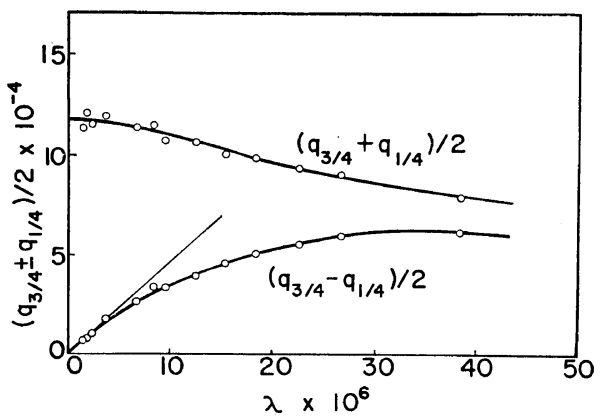

Figure 5. $\left[q_{3 / 4}(\lambda)+q_{1 / 4}(\lambda)\right] / 2$ and $\left[q_{3 / 4}(\lambda)-q_{1 / 4}(\lambda)\right] / 2$ vs. $\lambda$ for PCP fraction $f$ in MEK at $25.0^{\circ} \mathrm{C}$.

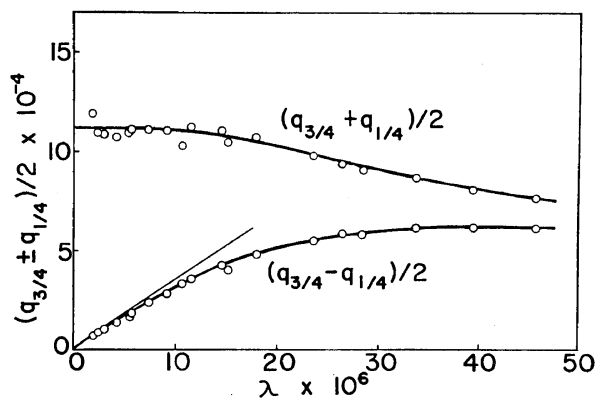

Figure 6. $\left[q_{3 / 4}(\lambda)+q_{1 / 4}(\lambda)\right] / 2$ and $\left[q_{3 / 4}(\lambda)-q_{1 / 4}(\lambda)\right] / 2$ vs. $\lambda$ for PCP fraction $F$ in MEK at $25.0^{\circ} \mathrm{C}$.

corresponding figures for the fraction $F$ are $9.06 \times 10^{4}$ and $9.23 \times 10^{4}$ from $\lambda q_{1 / 2}$ and $\lambda q_{1 / 4}$, respectively.

Values of $(1 / 2)\left(q_{1 / 4}+q_{3 / 4}\right)$ and $(1 / 2)\left(q_{3 / 4}-q_{1 / 4}\right)$ as functions of $\lambda$ for the fractions $f$ and $F$ are shown in Figures 5 and 6, respectively. In these graphs, we have no longer classified the data points for differing $c_{0}$ by different marks. Application of eq 7 yields $\bar{M}_{w}=(11.2 \pm 0.2) \times 10^{4}$ for the fraction $f$ and $\bar{M}_{w}=(11.8 \pm 0.2) \times 10^{4}$ for the fraction $F$, in excellent agreement with those obtained above from the curves for $q_{1 / 4}$ and $q_{1 / 2}$. This fact may be taken as a verification of the internal consistency of the present measurements. According to eq 8 , the initial slope of a plot of $(1 / 2)\left(q_{3 / 4}-q_{1 / 4}\right)$ against $\lambda$ should equal $\bar{M}_{z} \bar{M}_{w} / 4$. Application of this relation to the data in Figure 5, together with the $\bar{M}_{w}$ value for the fraction $f$, gives $\bar{M}_{z}=(12.5 \pm 0.7) \times 10^{4}$ for this fraction. The same procedure applied to Figure 6 yields $\bar{M}_{z}=(15.6 \pm 0.6) \times 10^{4}$ for the fraction $F$. The product $\bar{M}_{z} M_{w} / 4$ may also be
Table I. Average molecular weights and molecular weight ratios for PCP fractions $f$ and $F$

\begin{tabular}{lll}
\hline & \multicolumn{1}{c}{ Fraction $f$} & \multicolumn{1}{c}{ Fraction $F$} \\
\hline $\bar{M}_{n} \times 10^{-4}$ & $10.5 \pm 0.2(10.6)^{\mathrm{a}}$ & $9.15 \pm 0.08(8.8)^{\mathrm{a}}$ \\
$\bar{M}_{w} \times 10^{-4}$ & $11.2 \pm 0.2$ & $11.8 \pm 0.2(11.4)^{\mathrm{b}}$ \\
$\bar{M}_{z} \times 10^{-4}$ & $12.5 \pm 0.7$ & $15.6 \pm 0.6$ \\
$\bar{M}_{w} / \bar{M}_{n}$ & 1.07 & 1.29 \\
$\bar{M}_{z} / \bar{M}_{w}$ & 1.12 & 1.32
\end{tabular}

a From osmotic pressure measurements.

b From analysis of the interferometric sedimentation equilibrium pattern at $\lambda=4.87 \times 10^{-6}, c_{0}=$ $0.4031(\mathrm{~g} / \mathrm{d} l)$.

evaluated from the ordinate intercept of a plot of $\left(q_{3 / 4}-q_{1 / 4}\right) /(2 \lambda)$ against $\lambda$. Though the graphs are not shown here, we acertained that this method led to essentially the same values of $\bar{M}_{z}$ as those indicated above.

It was found that our experimental data were not accurate enough for either eq 9 or 10 to be applicable for the determination of $\bar{M}_{z+1}$. In fact, data points should have been obtained at lower $\lambda$ than treated here. However, it is doubtful whether this task can be done with accuracy in the currently available ultracentrifuges. Scholte, ${ }^{4}$ who also encountered a similar difficulty, had to be content with estimating only rough values for $\bar{M}_{z+1}$.

Table I summarizes the average molecular weights obtained from the variable $\lambda$ method and other conventional methods. It tells something of the accuracy and limitations of the variable $\lambda$ method, and also demonstrates that, as expected, the fraction $F$ has a broader molecular weight distribution than does the fraction $f$. Comparison of Observed and Calculated $q$ Curves

The function $q_{1 / 2}(\lambda)$ can be related to the molecular weight distribution, $g(M)$, of the sample by ${ }^{1-4}$

$$
q_{1 / 2}(\lambda)=\int_{0}^{\infty} \frac{\left(\lambda M^{2} / 2\right)}{\sinh (\lambda M / 2)} g(M) \mathrm{d} M
$$

Similar relations are available for $q_{1 / 4}$ and $q_{3 / 4}$. One may predict that the $q$ functions should be rather insensitive to molecular weight distribution, because $g(M)$ appears under the integral sign. At any rate, it seemed of interest to make a comparison between the observed and calculated $q$ functions in terms of eq 13 . 
Since the molecular weight ratios $\bar{M}_{w} / \bar{M}_{n}$ and $\bar{M}_{z} / \bar{M}_{w}$ for the fraction $f$ were found to be quite close to unity, we thought it reasonable to compare its $q$ curves with the ones calculated for a monodisperse sample that has an $M$ equal to its $\bar{M}_{w}\left(11.2 \times 10^{4}\right)$. One such comparison is displayed in Figure 3, in which the thin line represents the calculated values of $\lambda q_{1 / 2}$. There can be seen a considerable disparity between the calculated and observed curves both in the region of $\lambda$ encompassing the maxima and in the high molecular weight region. We may thus conclude that, though extracted after extensive fractionation, the fraction $f$ was still far from monodisperse in molecular weight.

The molecular weight ratios $\bar{M}_{w} / \bar{M}_{n}$ and $\bar{M}_{z} / \bar{M}_{w}$ for the fraction $F$ were found not to be very different from each other, both being close to 1.30. Hence it seemed adequate to try for the $g(M)$ of this fraction a logarithmic normal distribution which is defined by

$$
g(M)=\frac{\exp \left(-\beta^{2} / 4\right)}{\sqrt{\pi} M_{0} \beta} \exp \left\{-\frac{\left[\ln \left(M / M_{0}\right)\right]^{2}}{\beta^{2}}\right\}
$$

Here $M_{0}$ and $\beta$ are parameters which are related to $\bar{M}_{n}$ and $\bar{M}_{w}$ by

$$
\bar{M}_{w} / \bar{M}_{n}=\exp \left(\beta^{2} / 2\right), \quad \bar{M}_{n}=M_{0} \exp \left(\beta^{2} / 4\right)
$$

The thin line in Figure 4 shows the $\lambda q_{1 / 2}$ function computed for this $g(M)$ with $\bar{M}_{w}=11.8 \times 10^{4}$ and $\bar{M}_{w} / \bar{M}_{n}=1.30$. Its close agreement with the experimental data is to be noted, but it seems pertinent not to go beyond saying that the $g(M)$ of the fraction $F$ would not deviate much from a logarithmic normal type. For, as expected above, the $q$ functions should be rather insensitive to the details of $g(M)$.

\section{POLYISOBUTYLENE}

\section{Molecular Weights}

Figures 7 through 9 depict the various $q$ functions obtained for the PIB fraction in $\mathrm{EH}$ at $34.5^{\circ} \mathrm{C}$. Again, as required by theory, the data points for different initial concentrations merge on a single continuous curve. It is to be observed in Figure 8 that the plots for $\lambda q_{1 / 2}$ and $\lambda q_{1 / 4}$, especially that for the former, lack data points at $\lambda$ above $80 \times 10^{-6}$ and cannot be extrapolated without much uncertainty to the

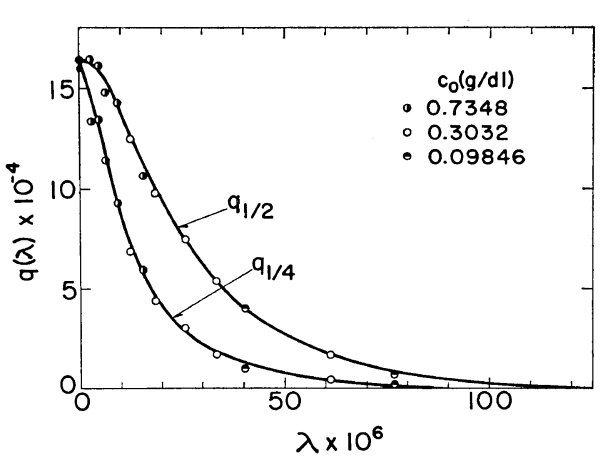

Figure 7. $q_{1 / 2}(\lambda)$ and $q_{1 / 4}(\lambda)$ vs. $\lambda$ for PIB (polyisobutylene) in EH (ethyl-n-heptanoate) at $34.5^{\circ} \mathrm{C}$. Small closed circle, $\bar{M}_{w}$ from light scattering; large closed circle, $\bar{M}_{w}$ from analysis of interferometric sedimentation patterns. Other types of circles correspond to different initial concentrations $c_{0}$.

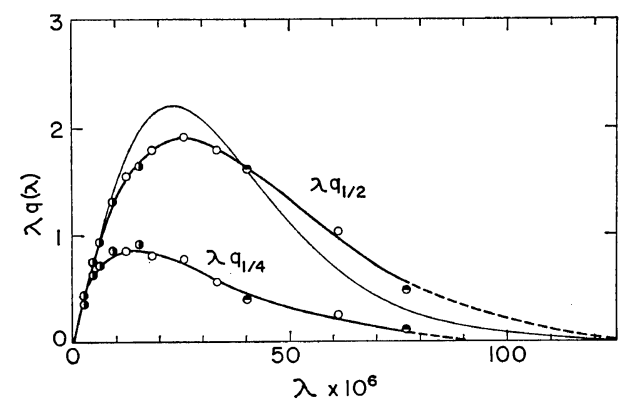

Figure 8. $\lambda q_{1 / 2}(\lambda)$ and $\lambda q_{1 / 4}(\lambda)$ vs. $\lambda$ for PIB in EH at $34.5^{\circ} \mathrm{C}$. The circles have the same meaning as in Figure 7. Thin line: $\lambda q_{1 / 2}(\lambda)$ calculated from eq 13 for a monodisperse sample with $M=16.4 \times$ $10^{4}$.

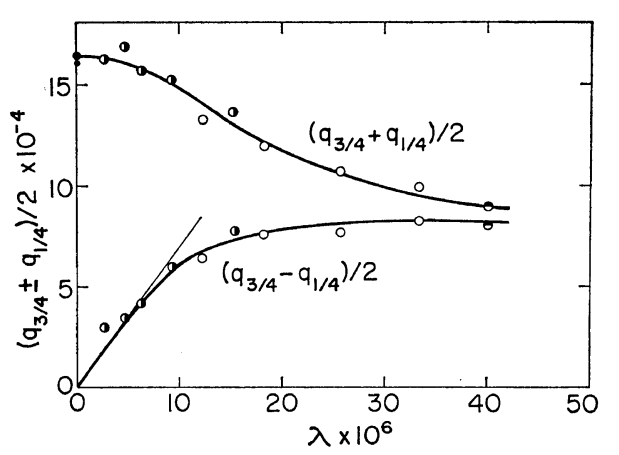

Figure 9. $\left[q_{3 / 4}(\lambda)+q_{1 / 4}(\lambda)\right] / 2$ and $\left[q_{3 / 4}(\lambda)-q_{1 / 4}(\lambda)\right] / 2$ vs. $\lambda$ for $\mathrm{PIB}$ in $\mathrm{EH}$ at $34.5^{\circ} \mathrm{C}$. The circles have the same meaning as in Figure 7. 
asymptotic zero level. This difficulty arose because the buoyancy factor for the system PIB$\mathrm{EH}$ was so small that the required large values of $\lambda$ were not attainable experimentally. A similar but less serious difficulty can also be seen in the plot for $q_{1 / 2}$ (Figure 7). The dashed lines in Figure 8 have been drawn so as to be consistent with the extrapolated curves in Figure 7. Because of the uncertainty they suffer, the $\bar{M}_{n}$ values from Figure 8 are correspondingly less accurate. We must also point to a marked scatter of the data points for $(1 / 2)\left(q_{3 / 4}-q_{1 / 4}\right)$ at the lowest $\lambda$ studied (Figure 9). This scatter makes it less certain to draw an initial tangent to the lower curve in Figure 9 and hence to estimate $\bar{M}_{z}$. Again, no convincing determination of $\bar{M}_{z+1}$ was feasible because of the lack of data points at lower $\lambda$ than treated here. Finally, the areas under the curves drawn in Figure 7 were 4.86 for $q_{1 / 2}$ and 2.54 for $q_{1 / 4}$. These are smaller by about 1.5 and $4 \%$ than the corresponding theoretical values.

The average molecular weights of the PIB fraction from the variable $\lambda$ method and other equilibrium methods are summarized in Table II. Despite the fact that the buoyancy factor

Table II. Average molecular weights and molecular weight ratios for the PIB fraction

\begin{tabular}{ll}
\hline $\bar{M}_{n} \times 10^{-4}$ & $14.9 \pm 0.6(15.3)^{\mathrm{a}}$ \\
$\bar{M}_{w} \times 10^{-4}$ & $16.4 \pm 0.4(16.0)^{\mathrm{b}}(16.4)^{\mathrm{c}}$ \\
$\bar{M}_{z} \times 10^{-4}$ & $17.4 \pm 0.8$ \\
$\bar{M}_{w} / \bar{M}_{n}$ & 1.10 \\
$\bar{M}_{z} / \bar{M}_{w}$ & 1.06 \\
\hline
\end{tabular}

a From osmotic pressure measurements. ${ }^{7}$

b From light scattering measurements. ${ }^{7}$

c Average of $\bar{M}_{w}$ from analysis of the interferometric sedimentation equilibrium patterns at $\lambda=$ $2.67 \times 10^{-6}, \quad c_{0}=0.7438(\mathrm{~g} / \mathrm{d} l) ; \lambda=4.67 \times 10^{-6}, \quad c_{0}=$ $0.7438(\mathrm{~g} / \mathrm{d} l)$; and $\lambda=12.39 \times 10^{-6}, c_{0}=0.3032(\mathrm{~g} / \mathrm{d} l)$.

of the PIB-EH system was so small that considerable uncertainty was thought unavoidable, the results from the variable $\lambda$ method are seen to be better than anticipated.

Comparison of Observed and Calculated $q$ Curves

The thin line in Figure 8 shows values of $\lambda q_{1 / 2}$ calculated from eq 13 for a monodisperse sample that has an $M$ equal to the $\bar{M}_{w}$ of the PIB studied. It deviates more appreciably from the corresponding observed values than was found in the case of PCP fraction $f$. Thus, though its $\bar{M}_{w} / \bar{M}_{n}$ and $\bar{M}_{z} / \bar{M}_{w}$ are quite close to unity (see Table II), the PIB fraction should still be too heterogeneous in molecular weight to be regarded as almost monodisperse. It should contain a considerable amount of lower molecular weight fractions, as judged from the disagreement of the observed and calculated curves in the high molecular weight region.

\section{CONCLUDING REMARKS}

The work reported in this paper has given further evidence for the conclusions reached by previous authors that $\bar{M}_{n}$ and $\bar{M}_{w}$ can be determined with reasonable accuracy by the variable $\lambda$ method of sedimentation equilibrium. The only other method now available for the evaluation of $\bar{M}_{z}$ is the classic Lansing-Kraemer method, ${ }^{9}$ which makes use of the sedimentation equilibrium curve at a single rotor speed. However, we have not tried this approach, because it did not seem of use for the narrow-distribution samples treated. In fact, it is well known both theoretically and experimentally that the concentration or concentration gradient distribution at sedimentation equilibrium does not reflect sensitively the polydispersity in molecular weight of a given sample. Thus, differing from Scholte's work, ${ }^{4}$ the accuracy of the $\bar{M}_{z}$ values obtained had to be left unchecked by another independent means.

It does not seem that the variable $\lambda$ method can be used routinely because it needs a great amount of experimental work including time and the cost of running the ultracentrifuge. However, we want to emphasize the fact that this method is not only founded rigorously on thermodynamic principles but also virtually the only practical approach for the absolute determination of $\bar{M}_{z}$. Such determinations should become imperative in establishing convenient relative methods for the estimate of $\bar{M}_{z}$.

\section{REFERENCES}

1. H. Fujita, J. Chem. Phys., 32, 1793 (1960). 


\section{Sedimentation Equilibrium}

2. H. Fujita, "Foundations of Ultracentrifugal Analysis," Wiley-Interscience, New York, N. Y., 1975 , p. 344.

3. H. W. Osterhoudt and J. W. Williams, J. Phys. Chem., 69, 1050 (1965).

4. Th. G. Scholte, J. Polym. Sci., Part A-2, 6, 91 (1968).

5. K. Hanafusa, A. Teramoto, and H. Fujita, $J$. Phys. Chem., 70, 4004 (1966).
6. T. G Fox and P. J. Flory, J. Amer. Chem. Soc., 73, 1909 (1951).

7. T. Matsumoto, N. Nishioka, and H. Fujita, $J$. Polym. Sci., Part A-2, 10, 23 (1972).

8. H. Murakami, T. Norisuye, and H. Fujita, Polymer J., 7, 248 (1975).

9. W. D. Lansing and E. D. Kraemer, J. Amer. Chem. Soc., 57, 1369 (1935). 